\title{
Human impacts on planetary boundaries amplified by Earth system interactions
}

Steven J. Lade ${ }^{1,2,3}$, Will Steffen ${ }^{1,2}$, Wim de Vries ${ }^{4,5}$, Stephen R. Carpenter ${ }^{6}$, Jonathan F. Donges ${ }^{1,7}$, Dieter Gerten ${ }^{7,8}$, Holger Hoff ${ }^{7,9}$, Tim Newbold ${ }^{10}$, Katherine Richardson ${ }^{11}$, Johan Rockström ${ }^{1,7}$

1. Stockholm Resilience Centre, Stockholm University, Stockholm, Sweden

2. Fenner School of Environment and Society, The Australian National University, Canberra, Australia

3. Bolin Centre for Climate Research, Stockholm University, Stockholm, Sweden

4. Environmental Systems Analysis Group, Wageningen University and Research, Wageningen, The Netherlands

5. Environmental Research, Wageningen University and Research, Wageningen, The Netherlands

6. Center for Limnology, University of Wisconsin, Madison, Wisconsin, USA

7. Potsdam Institute for Climate Impact Research, Member of the Leibniz Association, Potsdam, Germany

8. Geography Department, Humboldt-Universität zu Berlin, Berlin, Germany

9. Stockholm Environment Institute, Stockholm, Sweden

10. Centre for Biodiversity and Environment Research, Department of Genetics, Evolution and Environment, University College London, London, United Kingdom

11. Center for Macroecology, Evolution, and Climate, Globe Institute, University of Copenhagen, Copenhagen, Denmark

\section{ABSTRACT}

The planetary boundary framework presents a 'planetary dashboard' of humanity's globally aggregated performance on a set of environmental issues that endanger the Earth system's capacity to support humanity. While this framework has been highly influential, a critical shortcoming for its application in sustainability governance is that it currently fails to represent how impacts related to one of the planetary boundaries affect the status of other planetary boundaries. Here, we surveyed and provisionally quantified interactions between the Earth system processes represented by the planetary boundaries and investigated their consequences for sustainability governance. We identified a dense network of interactions between the planetary boundaries. The resulting cascades and feedbacks predominantly amplify human impacts on the Earth system and thereby shrink the safe operating space for future human impacts on the Earth system. Our results show that an integrated understanding of Earth system dynamics is critical to navigating towards a sustainable future.

\section{MAIN}

The planetary boundary framework assesses humanity's globally aggregated interference in nine Earth system processes compared to expert-estimated safe boundaries ${ }^{1}$. The nine processes are climate change, biogeochemical (nitrogen and phosphorus) flows, land-system change, freshwater use, aerosol loading, ozone depletion, ocean acidification, loss of biosphere integrity such as functional and genetic biodiversity, and introduction of novel entities such as toxic chemicals and plastics (Supplementary Table 1). Transgressing these boundaries threatens the capacity of the Earth system to maintain the Holocene-like state that allowed agriculture and complex human societies to develop. Since its inception in $2009^{2}$, the planetary boundary framework has been widely 
discussed $^{3}$, subject to critique $^{4,5}$ (see Methods), refined and updated ${ }^{1}$, and applied to policy at national ${ }^{6}$ and international ${ }^{7}$ scales.

The planetary boundaries interact, in that impacts on one planetary boundary can cause the Earth system to approach another planetary boundary ${ }^{1,2}$. For example, climate change may reduce the biosphere's ability to withstand human interference. The boundary for freshwater use is set at a level that should avoid threatening the integrity of freshwater ecosystems. While these interactions are broadly acknowledged, they are not visible in conventional representations of the planetary boundary framework ${ }^{1}$. Previous in-depth investigations of planetary boundary interactions have been limited to: (i) model-based studies of subsets of interactions, for example those involving the global carbon cycle ${ }^{8,9}$ or agricultural land-use decisions ${ }^{10-12}$; and (ii) surveys of which interactions are represented in global models ${ }^{13}$. Interactions between the Sustainable Development Goals, which the planetary boundary framework helped inform ${ }^{7}$, have been qualitatively assessed ${ }^{14-18}$ but a feedback analysis of the consequences of their interactions is also lacking.

Here, we surveyed and provisionally quantified interactions between almost the full set of planetary boundaries. We identified both biophysically- and human-mediated interactions, as demanded by a social-ecological view of the Earth system ${ }^{19}$. We split the boundary for biosphere integrity into boundaries for land, freshwater and ocean biosphere integrity, since the interactions that we identified frequently involved only one of these biosphere components and in recognition of the under-representation of aquatic dimensions in the current planetary boundary framework ${ }^{20}$. We then used a feedback model to calculate the consequences of these interactions for transgression of the planetary boundaries and the "safe operating space" for humanity within the Earth system. Our estimates of interaction strength and the subsequent model are highly simplified and in many cases highly uncertain representations of complex Earth system processes and should not be used to directly inform policy. Our goal is rather to stimulate discussion and research on the magnitude and consequences of planetary boundary interactions.

\section{RESULTS}

\section{A social-ecological survey of planetary boundary interactions}

For each Earth system process represented by the planetary boundaries, control variables indicate the degree to which humans are influencing that Earth system process (Fig 1A, vertical arrow). For each control variable, two reference values are identified: the boundary value, which delimits a conservative 'safe' range for the control variable (Fig 1A, green area); and a 'zone of uncertainty', a range of increasing risk beyond the boundary value (Fig $1 \mathrm{~A}$, yellow area).

Two types of interactions between components of the planetary boundary framework can occur (Fig $1 \mathrm{~A}$, arrows on left). First, changes in a control variable can lead to changes in the control variable of another planetary boundary. For example, land-system change can lead to carbon emissions that increase the atmospheric concentration of carbon dioxide, a control variable for the climate change planetary boundary. Here, the boundary value for climate change remains the same. Second, a change in a control variable can change the boundary value for another planetary boundary. For example, climate change may affect the amount of freshwater that can be safely extracted from terrestrial systems. We henceforth use 'interactions between planetary boundaries' and 'changes in a planetary boundary' to refer to both effects on control variable values and boundary values. 
We surveyed the literature for evidence on interactions between the planetary boundaries and data on the strength of the interaction (Methods). Taking a social-ecological system view of the Earth system, we sought interactions of the following types (Fig 1B).

- Biophysically-mediated interactions: Changes in a planetary boundary affect another planetary boundary through a biophysical mechanism. For example, land clearing (land system change planetary boundary) leads to carbon emissions (climate change planetary boundary).

- Reactive human-mediated interactions: A change in a planetary boundary can lead to a change in human behaviour that affects another planetary boundary. For example, decreased agricultural productivity due to climate change could lead via economic mechanisms to increased land clearing for agriculture (land system change planetary boundary) at the globally aggregated scale.

- Parallel human drivers: Human impacts on a planetary boundary are often associated with subsequent impacts on another planetary boundary due to their common drivers. For example, land clearing (land system change planetary boundary) is often followed by increased freshwater use and biogeochemical flows, due to the common driver of agriculture that causes land clearing, freshwater use and biogeochemical flows.

While the interactions can be biophysically or human-mediated, all interactions are ultimately caused by direct human impacts on a planetary boundary that then trigger subsequent interactions.

The biophysically-mediated interactions that we identified (Supplementary Methods) include: impacts of surface climate warming on land, freshwater and ocean biosphere integrity and stratospheric ozone; impacts of climate change, land-system change and aerosol loading via changed rainfall runoff patterns on freshwater availability and biogeochemical flows; eutrophication in freshwater and ocean systems due to nutrient inputs and freshwater extraction; and climate change and ocean acidification due to carbon emissions from deforestation, changes in uptake by terrestrial, marine and freshwater ecosystems, and radiative forcing from aerosol loading. The parallel human drivers involved carbon emissions that lead to both climate change and ocean acidification, emission of ozone-depleting substances that are also greenhouse gases, and the food-energy-water nexus: clearing of land for agriculture is usually followed by application of fertilisers and freshwater; fertilisers and freshwater use involve carbon emissions from electricity generation; and electricity generation often involves water use. The reactive human-mediated interactions that we identified related to increased agricultural activity in response to loss of protein from freshwater or marine fisheries, and increased carbon emissions to treat or generate water in response to declines in surface water quality.

The interactions ranged from well-characterised (such as the effect of atmospheric carbon dioxide on ocean acidification) to highly unconstrained (such as interactions involving biosphere integrity). Our survey and estimates of interaction strengths (summarised quantitatively in Supplementary Table 2 and graphically in Fig. 2) should be treated as an initial, speculative attempt at characterising these interactions. We welcome further work to identify additional interactions beyond those we listed and to better constrain their interaction strengths.

\section{The planetary boundaries are densely interconnected}

Our survey found evidence for over half the possible interactions between different pairs of planetary boundaries (52 out of 90; Supplementary Table 2). We could quantify an interaction for 35 
of these interactions (Supplementary Table 2 and Supplementary Methods). This dense network of interactions between the planetary boundaries is unsurprising, since the Earth system is known to be tightly interconnected ${ }^{21,22}$. Only six biophysically-mediated interactions are attenuating (negative) interactions (Supplementary Table 2), where greater disruption of the Earth system process corresponding to one planetary boundary leads to less disruption of the Earth system process corresponding to another planetary boundary; once human-mediated interactions are accounted for only four interactions are net attenuating (Supplementary Table 2; bordered links in Fig 2A). We expect that interactions between the planetary boundaries therefore mostly amplify human impact on the Earth system, a hypothesis that we test below.

The climate change and biosphere integrity planetary boundaries were identified by Steffen et $a l .{ }^{1}$ as the two 'core' planetary boundaries, which are regulated by processes corresponding to the other 'non-core' planetary boundaries. Our survey supports their status as core elements within the network of planetary boundary interactions. First, the core boundaries receive and originate greater total strength of biophysically-mediated interactions than other planetary boundaries (Supplementary Table 3). Second, a force-directed network diagram ${ }^{23}$, which arranges nodes with stronger interactions closer together, places the two core boundaries near the centre of the diagram (Fig 2B). Our survey also found evidence for interactions among non-core boundaries (12 interactions, of which we could quantify 6; Supplementary Table 2), indicating that the interactions among the planetary boundaries are more complex than a hub-and-spoke pattern between the core and non-core boundaries.

\section{Interactions have amplified human impacts on planetary boundaries}

We constructed a simple linear feedback model to illustrate possible consequences of interactions between the planetary boundaries (Methods). Since specific outputs of the model are based on the often highly unconstrained estimates of interaction strengths, we use it only to illustrate possible consequences of the interactions; it should not be used to quantitatively inform policy decisions.

We first used our model to estimate how much of the current value of each control variable is due to direct human impacts and how much is due to the propagation of direct impacts via interactions (Fig 3). Over all planetary boundaries, biophysically-mediated interactions contributed $31 \%$ of the total current values of normalised control variables compared to $40 \%$ for direct human impacts, $28 \%$ for parallel human drivers and $1 \%$ for reactive human-mediated interactions (Methods). Biophysicallymediated interactions have therefore almost doubled direct human impacts on the planetary boundaries (or nearly $50 \%$ increase over direct human impacts and parallel human drivers combined). Reducing the strengths of biophysically-mediated interactions could therefore significantly reduce future impacts on the planetary boundaries. These interactions, however, reflect basic biophysical mechanisms such as the radiative forcing contributed by atmospheric carbon dioxide emitted by land clearing for agriculture, or nutrient overuse leaching into freshwater systems leading to eutrophication. Modifying these interactions would require costly, difficult to govern ${ }^{24}$ and possibly counterproductive ${ }^{9,12}$ geoengineering.

Biogeochemical flows were controlled mainly by parallel human drivers (Fig 2A; Supplementary Table 2); specifically, nutrient application on cropland frequently occurred subsequent to clearing that land from forest ${ }^{25}$. Freshwater use, ocean acidification and climate change had mixed contributions from biophysical interactions, parallel human drivers, and direct human impacts (Fig 
3). Of the planetary boundary interactions that we identified, some of the parallel drivers are perhaps the most amenable to intervention. For example, better nutrient management could break the link between land clearing for agriculture and (in the global aggregate) excessive nutrient application that has led to anthropogenic biogeochemical flows exceeding the planetary boundary.

Reactive human-mediated interactions, such as degradation of freshwater biosphere integrity leading to increased carbon emissions from water purification or desalinisation, had relatively small globally-aggregated contributions to interactions in our analysis (Fig 3). Interactions of this type can arise from unintended consequences, such as an increase in agricultural activity in response to the construction of dams that degrade freshwater fisheries ${ }^{26}$, and therefore difficult to anticipate. Policy instruments could also create new interactions via economic mechanisms ${ }^{27}$. Some interactions may only manifest after very severe transgressions of planetary boundaries that still have not yet been experienced, for example severe climate change. We encourage future work to better capture human-mediated interactions.

\section{A safe operating space for future sustainability governance}

The planetary boundaries delimit a 'safe operating space' for humanity on planet Earth ${ }^{28}$. Remaining outside this safe operating space could lead away from a safe Stabilised Earth trajectory to an unsafe Hothouse Earth trajectory for the Earth system ${ }^{29}$. We characterise the goal of future sustainability governance as navigating back towards the safe operating space and a Holocene-like state of the Earth system.

For the analysis of interactions in this paper, we define the safe operating space as those combinations of human impacts on the planetary boundaries that cause no planetary boundary to be transgressed. We include in the category of 'human impacts' both direct human impacts and impacts mediated by human behaviour (of parallel and reactive types). Since most interactions between planetary boundaries are amplifying (Fig 2A), we expect incorporating knowledge about interactions to shrink the safe operating space for human impacts. Such shrinkage would reduce Earth system resilience and further complicate the challenge of Earth system governance in the Anthropocene.

For an initial estimate of the safe operating space with interactions taken into account, we set all control variables at their planetary boundaries and used the model to back-calculate the combination of human impacts that would lead to those control variable values (see Methods). We found that along most planetary boundaries, interactions do indeed shrink the safe operating space (Fig. 4). The largest exception is freshwater use: since climate change will increase precipitation, the safe level of globally aggregated freshwater use could increase, though this result would depend strongly on the location of increased rainfall.

This method, however, leads to negative edges of the safe operating space for the ocean and freshwater biosphere integrity planetary boundaries (Fig 4). If all other control variables are at their planetary boundaries, human actions that massively improve ocean and freshwater biosphere integrity would therefore be necessary to stay within the safe operating space. This result occurs because, under the assumptions of the model, either the biogeochemical flows or freshwater use control variables at their planetary boundary pushes the freshwater biosphere integrity control variable to its planetary boundary. Their additive effects plus impacts from climate change and land 
system change push freshwater biosphere integrity well beyond its planetary boundary. Similar reasoning holds for ocean biosphere integrity, with climate change and ocean acidification causing the greatest impacts. Actions to improve freshwater and ocean biosphere integrity such as transplantation of nursery-grown coral ${ }^{30}$, ocean plastic removal, freshwater sediment dredging to remove nutrient loading ${ }^{31}$ or fish restocking ${ }^{31}$, are conceivable on a small scale but likely prohibitively expensive at the scale demanded by our analysis.

\section{Trade-offs within the safe operating space}

We expect that trade-offs between the planetary boundaries, generated by their predominantly amplifying interactions, could be exploited to navigate back to the safe operating space. For example, the interactions described above suggest that massive global action to improve freshwater biosphere integrity could be avoided by reducing freshwater use or anthropogenic contributions to biogeochemical flows, or a combination of both, below their respective boundary values.

Here we illustrate trade-offs in the safe operating space between two broad categories of human impacts on the Earth system: agricultural activity and carbon emissions. We represented agricultural activity by applying direct impacts on land-system change (such as land clearing), which via the model's parallel human drivers result in human impacts from agricultural activity on freshwater use and biogeochemical flows. We represented carbon emissions (including emissions from agriculture) by applying direct impacts on climate change, which via the model's biophysically-mediated interactions result in impacts on ocean acidification. We used our model to estimate the consequences of these impacts on the full set of planetary boundaries included in our model. For different combinations of agricultural activity and carbon emissions, we calculated how many planetary boundary values and zones of uncertainty would be transgressed (Fig 5).

Without interactions, the safe operating space is bounded by the planetary boundaries for biogeochemical flows and climate change (Fig 5 , green dashed line), since agricultural activity and carbon emissions cause these boundaries to be crossed first. With interactions, the size of the safe operating space shrinks substantially (Fig 5 , green area). The first planetary boundary to be transgressed as agricultural activity or carbon emissions are increased is freshwater biosphere integrity, though several other planetary boundaries (Fig 5, darker yellow areas) and zones of uncertainty (Fig 5, red areas) are transgressed soon thereafter. These results show that interactions can lead to cascading transgressions of multiple planetary boundaries. Similar cascades were recently suggested to potentially lead to an unsafe Hothouse Earth trajectory for the Earth system ${ }^{29}$.

As well as shrinking in size, the shape of the safe operating space in this graphical representation changes after interactions are taken into account from square to roughly triangular (Fig 5, green area). The triangular shape leads to trade-offs: if carbon emissions are low then high levels of agricultural activity are safe, and vice versa, but high levels of both agricultural activity and carbon emissions cannot be safely maintained. This shape of the safe operating space is similar to that found previously in a conceptual model ${ }^{32}$. In our model, if agricultural activity is low then the safe level of carbon emissions is even higher than pre-interaction levels, due to the masking effects of aerosol loading. 


\section{Navigating towards a safe operating space}

The current state of the Earth system as represented by the planetary boundaries is well outside the safe operating space for human impacts (Fig 5). Actions that navigate the Earth system back towards the safe operating space are urgently needed. Due to biophysical, economic and other social interactions, however, policies addressing a specific planetary boundary will often lead to impacts on other planetary boundaries ${ }^{27}$.

We investigated two climate mitigation measures that involve changes in agricultural activity: Largescale bio-energy production with carbon capture and storage (BECCS), where carbon dioxide from the combustion of rapidly growing crops is geologically sequestered, and a global transition to lowmeat diets. We used published scenarios to estimate the effects of these measures (Supplementary Methods). They correspond to a small subset of the agricultural practices that could be used to implement BECCS and a small subset of possible food system transitions towards a low-meat diet, and therefore should be considered only as illustrative.

The direct impact of BECCS through carbon draw-down could substantially reduce impacts of carbon emissions $^{11,33}$ (Fig 5). The large-scale biomass plantations required for BECCS, however, lead to increased agricultural activity that, via interactions, lead to carbon emissions that counter the reductions achieved by BECCS. The result is, under the assumptions of our model, a trajectory at best parallel to the safe operating space (Fig 5). Some studies even suggest that carbon emissions from land use change (an interaction between the land system change and climate change planetary boundaries) could outweigh carbon draw-down leading to net positive carbon emissions from $\mathrm{BECCS}^{34,35}$. Furthermore, our simple model underestimates impacts on the freshwater use, land biosphere integrity and biogeochemical flows planetary boundaries (Supplementary Methods), because BECCS will likely involve more intensive agriculture than the simple globally and historically aggregated interactions assumed in our model.

While the direct impact of low-meat diets on carbon emissions may be smaller than large-scale BECCS, low-meat diets typically lead to reduced agricultural activity and a trajectory moving towards the safe operating space (Fig 5). Reduced agricultural activity triggers interactions that further lower carbon emissions (Fig 2A; Supplementary Table 2). Our results reinforce that low-meat diets, alongside other transformations of the food system ${ }^{36}$, are an important strategy for navigating back towards the safe operating space for humanity in the Earth system ${ }^{37}$.

\section{DISCUSSION}

Our results offer three key findings for policymakers. First, interactions are crucial to understanding the planetary boundaries and humanity's impacts upon them. For example, we calculated that biophysically-mediated interactions have almost doubled direct human impacts on the planetary boundaries. Second, most interactions we found were amplifying, meaning that impacts on one planetary boundary lead to increased impacts on other planetary boundaries (Fig 2). Cascading of human actions through multiple components of the Earth system complicates governance of the Earth system. On the other hand, these interactions offer substantial scope for synergies: if impacts on one planetary boundary are decreased, impacts on other planetary boundaries may also lessen. Our survey of planetary boundary interactions (Fig 2) offers a roadmap for identifying where these synergies lie. Third, interactions between planetary boundaries lead to trade-offs between the 
boundaries (Fig 5). For example, interactions between agricultural activity and carbon emissions mean that high levels of both cannot be maintained. On the other hand, these trade-offs offer humanity some freedom in choosing how to navigate to a safe operating space.

We caution that while our model can yield insight into consequences of interactions, the interaction strength estimates it uses are often poorly constrained and are globally aggregated. Some planetary boundaries are highly spatially heterogenous ${ }^{1}$, and the distribution of humanity's contributions to globally aggregated boundaries such as climate change is also highly heterogeneous, so we expect that many planetary boundary interactions are also spatially and socio-culturally heterogeneous. Our model in its current form should therefore not be used for policy design, though our methods could be adapted to complement empirical assessments of regional safe operating spaces ${ }^{38-40}$. The planetary boundary framework only captures limited aspects of changes in the Earth system, and our study of interactions can therefore only capture a limited number of Earth system processes. Our model accounts for feedbacks between planetary boundaries, but does not account for nonlinearities such as interactions that activate after a control variable reaches some threshold, dynamics such as time lags, or interactions of higher order than pairwise such as the multiplicative effects of climate change and land-system change on biodiversity loss ${ }^{41}$. These shortcomings offer promising avenues for future research towards the challenge of navigating back towards a safe operating space for humanity. Our approach for modelling interactions could also be applied to other frameworks for global sustainability, such as Raworth's 'doughnut'42 or the Sustainable Development Goals, for which understanding interactions is also critically important ${ }^{14-16,18,43,44}$.

The original planetary boundary framework ${ }^{2}$ has been used both as a high-level policy reference illustrating humanity's performance on environmental issues of global concern and as an object for scientific and policy-based scrutiny and refinement. We offer our survey of planetary boundary interactions to policymakers and the scientific community in the same spirit: as a summary of current scientific knowledge, a call for future research to better characterise interactions, and as a framework to prompt policy discussions and planning towards a sustainable future.

\section{METHODS}

\section{Planetary boundaries}

We included in our analysis of interactions all planetary boundaries except the boundary for introduction of novel entities, which is difficult to systematically assess at present. As in the previous versions of the framework ${ }^{1,2}$, the planetary boundaries describe limits that should not be transgressed to maintain the Earth system in a Holocene-like state.

We retained the framework presented by Steffen et al. ${ }^{1}$ as closely as possible, while recognising that many control variables are imperfect indicators of the underlying Earth system processes ${ }^{1,2}$. We found it necessary however to split the planetary boundary for biosphere integrity into planetary boundaries for land, freshwater and ocean biosphere integrity. Interaction mechanisms involving terrestrial and aquatic biospheres differ significantly. While the marine and freshwater biospheres are more similar, some interactions such as the effects of freshwater use and ocean acidification are significantly different in magnitude between these two spheres. 
We did not separate the biogeochemical flow boundary into nitrogen $(\mathrm{N})$ and phosphorous $(\mathrm{P})$ flows since it would involve an increase in model complexity that does not affect model results, for the following reasons. In this de-aggregated model, $\mathrm{N}$ and $\mathrm{P}$ inputs would occur in direct proportion due to their shared driver (agricultural activity). These direct inputs and increased runoff due to increased precipitation, which we expect affect $\mathrm{N}$ and $\mathrm{P}$ equally, are the only factors whose strength we estimate that affect $\mathrm{N}$ and $\mathrm{P}$ flows (Supplementary Table 2). Furthermore, their current normalised control variables (see below) have almost identical values (Supplementary Table 1). Therefore, while the mechanisms by which $\mathrm{N}$ and $\mathrm{P}$ affect other planetary boundaries are different, their normalised control variables could mathematically be interchanged without affecting the result. The relative concentrations of $\mathrm{N}$ and $\mathrm{P}$ may vary between terrestrial application and those in freshwater and marine ecosystems, but these concentrations do not directly enter our model. Splitting the biogeochemical flows boundary, and the biosphere integrity control variables into more specific features, may be necessary in future work.

The planetary boundaries framework has been subject to some critique. Criticisms have included that the global scale of the planetary boundary framework distracts from managing local-scale issues such as biodiversity loss or water overuse and that global tipping points are unlikely for processes that operate mostly at local or regional scales such as loss of biodiversity ${ }^{4,5,45,46}$. Responses have included that a planetary boundary does not necessarily imply a tipping point and that the framework is a synthesis of anthropogenic impacts significant at the global scale that was never intended to replace local-scale management approaches ${ }^{1,47,48}$. The purpose of this article is not to contribute to these debates. We note however that our modelling framework (see section 'Control theory framework' below) does not assume any tipping dynamics at or near a planetary boundary.

\section{Normalised control variables}

Let $X$ be the planetary boundary control variable, $X_{0}$ its pre-industrial values, and $X_{\mathrm{PB}}$ its boundary value. The planetary boundaries all represent different Earth system processes with different physical units. To compare the strengths of interactions between planetary boundaries, we first define normalised control variables

$$
x=\frac{X-X_{0}}{X_{\mathrm{PB}}-X_{0}} .
$$

Lower case symbols hereafter denote normalised control variables and upper case symbols denote un-normalised control variables. Under Eq. (1), a normalised control variable has value 0 at preindustrial conditions and 1 at its boundary value. Values below 0 and above 1 are possible and correspond to a control value improved beyond pre-industrial and worsened beyond the boundary, respectively. Changes in the un-normalised control variable $(X)$ or the boundary value $\left(X_{\mathrm{PB}}\right)$ will cause a change in the normalised control variable.

We calculated the normalised values for the current control variable values and zones of uncertainty for the planetary boundaries considered here (Supplementary Table 1). Where there were two or more control variables for a planetary boundary (such as for climate and biogeochemical flows), we averaged the normalised values to give a single normalised control variable value. For the biogeochemical flows planetary boundary we used the two control variables directly subject to human action, "P flow from fertilisers to erodible soils" and "industrial and intentional biological fixation of $\mathrm{N}$ ", omitting the control variable "P flow from freshwater systems into the ocean" due to 
its highly uncertain value ${ }^{49}$. For the stratospheric ozone planetary boundary we used total column ozone (which is dominated by stratospheric ozone), averaged over mid-latitudes as assessed by the World Meteorological Association ${ }^{50}$. This mid-latitude measure is more indicative of global ozone depletion and leads to more significant Earth system interactions than polar ozone depletion. Since there are no available estimates of control variable values for the here newly defined freshwater and ocean biosphere integrity boundaries, we estimated their normalised control variable values using an indirect method (see below).

\section{Literature survey}

We surveyed the literature for interactions between the planetary boundaries. For each interaction, we performed a search on Scopus (last performed 24 June 2019) with search term "[PB1] [PB2] global $^{* \prime}$ in Title, where [PB1] and [PB2] were set according to:

- Climate change: "climate change" OR "radiative forcing" OR "greenhouse gas*" OR "carbon dioxide"

- Biosphere integrity (land): biodiversity OR "ecosystem health"

- Biosphere integrity (freshwater): freshwater OR river* OR lake* OR inland

- Biosphere integrity (ocean): "biological pump" OR "coral reefs" OR fish* OR "marine biodiversity"

- Land-system change: "land-system change" OR "land cover" OR deforestation OR "habitat loss"

- Biogeochemical flows: nitrogen OR phosphorus OR fertiliser OR fertilizer

- Ocean acidification: "ocean acidification"

- Freshwater use: precipitation OR runoff OR "water cycle" OR "hydrological cycle" OR "water consumption"

- Aerosol loading: aerosol*

- Stratospheric ozone depletion: "stratospheric ozone"

We reviewed abstracts and where appropriate read manuscripts to identify those articles that assessed a globally aggregated strength of the interaction. We sought interactions that operate on policy-relevant time scales of 100 years. Where the search yielded no useful results, we expanded the search by: (a) changing the search to include title, abstract and keywords; (b) changing the search term to "[PB1] [PB2]" in Title. In all searches, we only examined results from publications after the year 2000 .

We sought representative literature for each interaction; exhaustive surveys of each interaction and analyses of their uncertainties were beyond the scope of this article. We supplemented the search with our own knowledge of the literature. In a small number of cases, we constructed our own estimates of interaction strengths using published data.

For the freshwater and ocean biosphere integrity planetary boundaries that we introduce in this article, control variables have not yet been defined. For interactions involving these planetary boundaries, we relied on assessments of the levels at which various ecosystem functions will be significantly affected. These ecosystem functions include production of fish biomass, the marine biological carbon pump (ocean only), depletion of aragonite-forming organisms (ocean only), and water quality; see the individual interactions in Supplementary Methods for further detail. 


\section{Estimation of interaction strengths}

For each interaction, we label the originating normalised control variable for the interaction as $x$ and the receiving normalised control variable for the interaction as $y$, that is, the interaction is $x \rightarrow y$. For each interaction, we estimated the normalised interaction strength defined by

$$
s=\frac{\Delta y}{\Delta x}
$$

where $\Delta x$ is the change in the normalised control variable $x$ that leads to a change $\Delta y$ in the normalised control variable $y$. The Supplementary Methods describe the interactions we identified and our estimations of the interaction strengths. Supplementary Table 2 summarises our estimates of normalised interaction strengths.

Changes in both an un-normalised control variable value and in a boundary value can cause changes in a normalised control variable value (see Eq. 1). Where data are available on changes in normalised control variable values, Eq. (2) can be used directly to estimate the normalised interaction strength. In the list below, Eq. (1) and (2) have been used to derive equivalent expressions to expedite calculations in cases where only changes in un-normalised control variables or boundary values are directly available. These equations hold for cases where:

- A change in un-normalised control variable $\Delta X$ causes a change in another un-normalised control variable $\Delta Y$ (but $X_{\mathrm{PB}}$ and $Y_{\mathrm{PB}}$ are fixed),

$$
s=\frac{\Delta Y}{\Delta X} \frac{X_{P B}-X_{0}}{Y_{P B}-Y_{0}} .
$$

- A change in un-normalised control variable $\Delta X$ causes a change in the boundary value of another planetary boundary from $Y_{\mathrm{PB}}$ to $Y_{\mathrm{PB}}^{\prime}$ (but $X_{\mathrm{PB}}$ and $Y$ are fixed),

$$
s=\left(\frac{Y-Y_{0}}{Y_{\mathrm{PB}}^{\prime}-Y_{0}}-\frac{Y-Y_{0}}{Y_{\mathrm{PB}}-Y_{0}}\right) \frac{X_{\mathrm{PB}}-X_{0}}{\Delta X} .
$$

We expect that this type of interaction would also change the zone of uncertainty but we do not model this effect here.

- As for equation (4), but where evidence on the change in the originating control variable is available in normalised units $\Delta x$

$$
s=\left(\frac{Y-Y_{0}}{Y_{\mathrm{PB}}^{\prime}-Y_{0}}-\frac{Y-Y_{0}}{Y_{\mathrm{PB}}-Y_{0}}\right) \frac{1}{\Delta x} .
$$

Where the planetary boundary has more than one control variable, we looked for interactions involving either control variable. For example, for the climate change planetary boundary we looked for interactions involving either carbon dioxide concentrations or radiative forcing.

Some interaction strengths are 1 because some planetary boundaries are defined by the effect of that boundary's transgression on another planetary boundary. For example, the ocean acidification planetary boundary is defined as that at which the functioning of marine ecosystems is compromised, that is, when the marine biosphere integrity planetary boundary is transgressed.

We did not assess Earth system feedbacks that involve only one planetary boundary, for example, the long-wave radiation into space that partially stabilises the Earth's climate against temperature increases. 


\section{Control theory framework}

Control theory studies how feedbacks modify the operation of systems. Engineering commonly uses control theory to design feedbacks that achieve desired system behaviour ${ }^{51}$. The feedbacks associated with environmental management, such as fishery quota setting in response to stock assessments, can also be expressed in a control theory framework ${ }^{52}$. Here, we use a control theory framework, but without any feedback design, to calculate the effects of interactions between the planetary boundaries. In the following, bold lower-case symbols denote vectors of the relevant quantities for the planetary boundaries considered here.

The state of the normalised control variables $\mathbf{x}$ without feedbacks is simply given by the direct human impacts $\mathbf{d}$ (impacts that do not result from changes in another planetary boundary) (Box 1 ). With feedbacks active we first calculate the human impacts $h$, which are comprised of: direct human impacts $\mathbf{d}$; plus impacts arising from changes in normalised control variables $\mathbf{x}$ mediated by reactive human mechanisms $\mathbf{R}$; plus parallel impacts from reactive interactions and direct drivers on other planetary boundaries $\mathbf{P}(\mathbf{d}+\mathbf{R} \mathbf{x})$ :

$$
\mathbf{d}+\mathbf{R} \mathbf{x}+\mathbf{P}(\mathbf{d}+\mathbf{R} \mathbf{x})=\mathbf{h} .
$$

The values of the normalised control variables $\mathbf{x}$ are comprised of human impacts $\mathbf{h}$ plus impacts arising from changes in other normalised control variables $\mathbf{x}$ mediated by biophysical mechanisms, $\mathbf{B}$, giving

$$
\mathbf{h}+\mathbf{B} \mathbf{x}=\mathbf{x} .
$$

Solving equations (6) and (7) by eliminating $h$, we find that interactions have amplified initial direct impacts according to

$$
x=[I-(B+R+P R)]^{-1}(I+P) d,
$$

where $\mathbf{I}$ is the identity matrix.

This approach assumes that the control variables $\mathbf{x}$ have reached equilibrium in response to the current values of the direct impacts $\mathbf{d}$. Many components of the Earth system, such as the carbon cycle, contain transient dynamics and time lags that our model cannot capture. Furthermore, our estimations of different interaction strengths (Supplementary Methods) are based on a variety of time periods due to data constraints. The model is also linear and therefore does not account for nonlinear interactions, for example that only activate after a control variable reaches some threshold, nor does the model generate any of the tipping point dynamics that are associated with transgressing some of the planetary boundaries ${ }^{1}$.

We use this linear, equilibrium model as a first attempt to quantify how interactions between planetary boundaries affect the relationship between direct human impacts and the transgression of planetary boundaries. Incorporating dynamics and non-linearities would better represent the behaviour of the Earth system and potentially be more useful for governance and is a promising avenue for future work. Adding such further detail to the model would however come with the cost of requiring more information to be gathered to characterise each interaction.

\section{Inferring the normalised control variable values for the ocean and freshwater biosphere integrity planetary boundaries}

Control variables for the ocean and freshwater biosphere integrity boundaries have not previously been empirically estimated. The first step of our analysis was to estimate values for the current 
normalised values for these boundaries based on their interactions with other boundaries. We assume that these biosphere integrity boundaries only experience human impact through their interactions with other boundaries, so their direct human impacts are zero. Even under this conservative assumption, we calculate below that marine and freshwater biosphere integrity is strongly degraded. Future work could incorporate direct human impact on aquatic systems, for example through fisheries or dams.

We first outline our logic for calculating these control variable values without mathematical formalism. Since the biosphere integrity boundaries are significantly affected by other boundaries, and we know the control variable values of those planetary boundaries and the strengths of their interactions with the biosphere integrity boundaries, we can therefore estimate the biosphere integrity control variable values. For example, freshwater biosphere integrity experiences impacts from climate change, land system change, biogeochemical flows and freshwater use (Supplementary Table 2) which have current normalised control variables of 2.0, 1.5, 2.3 and 0.65, respectively (Supplementary Table 1). For the normalised control variable for freshwater biosphere integrity, this logic gives a value $2.0 * 0.38+1.5 * 0.08+2.3 * 1+0.65 * 1=3.8$.

Formally, we used the following reasoning to ensure consistency with the control theory framework. Let $b$ be the set of the two unknown biosphere integrity planetary boundaries and $\bar{b}$ be the complementary set of the other planetary boundaries. We seek estimates of the control variable values $\mathbf{x}_{b}$. Defining

we re-write equation (8) as

$$
A=(I+P)^{-1}[I-(B+R+P R)]
$$

$$
\mathbf{A} \mathbf{x}=\mathbf{d} \text {. }
$$

We pick out the rows $b$ of this vector equation corresponding to the unknown biosphere integrity planetary boundaries (in the ordering given in Supplementary Table 2, $b=\{3,4\}$ ):

$$
\mathbf{A}_{b, *} \mathbf{x}=\mathbf{d}_{b}=\mathbf{0} \text {. }
$$

Here, $\mathbf{A}_{m, n}$ denotes the submatrix of $\mathbf{A}$ formed by those elements with row numbers in $m$ and column numbers in $n$; the placeholder ' $*$ ' is understood to refer to all columns. We subtract the terms on the left-hand side for which $\mathbf{x}$ is known (terms involving $\mathbf{x}_{\bar{b}}$ ) over to the right-hand side,

$$
\mathbf{A}_{b, b} \mathbf{x}_{b}=-\mathbf{A}_{b, \bar{b}} \mathbf{x}_{\bar{b}}
$$

which we then solve for the unknown values $\mathbf{x}_{b}$ :

$$
\mathbf{x}_{b}=-\left[\mathbf{A}_{b, b}\right]^{-1} \mathbf{A}_{b, \bar{b}} \mathbf{x}_{\bar{b}} .
$$

Using this equation we estimated the following current values for the normalised biosphere integrity control variables:

- Freshwater biosphere integrity: 3.8, which is over three times the planetary boundary. This value is plausible considering the considerable stress freshwater ecosystems are currently under ${ }^{53}$ from biogeochemical flows and freshwater extraction.

- Ocean biosphere integrity: 1.4 , which is over the safe planetary boundary at the globally aggregated scale. This is plausible considering the considerable stress marine ecosystems are experiencing from ocean acidification and climate change.

Using these values ensures consistency when interactions with the other planetary boundaries are applied. As argued above, they are also plausible values for the boundaries. We do not assign any 
upper end to the zones of uncertainty for these two boundaries, in the absence of information to do so.

\section{Consequences of interactions between the boundaries}

Rearranging Eq. (8) gives

$$
\mathbf{x}=\mathbf{d}+\mathbf{B} \mathbf{x}+\mathbf{R} \mathbf{x}+\mathbf{P} \mathbf{d}+\mathbf{P} \mathbf{R} \mathbf{x} .
$$

We therefore compared the different contributions to the current values of planetary boundary control variables, $\mathbf{x}$, by using: $\mathbf{d}$ for the contributions of direct human impacts; $\mathbf{B} \mathbf{x}$ for the contributions of biophysically-mediated interactions; $\mathbf{R} \mathbf{x}$ for the contributions of reactive humanmediated interactions; and $\mathbf{P} \mathbf{d}+\mathbf{P} \mathbf{R} \mathbf{x}$ for the contributions of parallel human drivers. Direct human impacts, $\mathbf{d}$, were calculated by rearranging Eq. (8) to give

$$
\mathbf{d}=(\mathbf{I}+\mathbf{P})^{-1}[\mathbf{I}-(\mathbf{B}+\mathbf{R}+\mathbf{P} \mathbf{R})] \mathbf{x} \text {. }
$$

To estimate the total contribution of each interaction type to the current values of the normalised control variables, we compared the sums over all elements of $\mathbf{B} \mathbf{x}, \mathbf{R} \mathbf{x}, \mathbf{P} \mathbf{d}+\mathbf{P} \mathbf{R} \mathbf{x}$ and $\mathbf{d}$ to the sum over all elements of $\mathbf{x}$. We re-aggregated the biosphere integrity boundaries by averaging across the three biosphere integrity elements for each vector.

\section{Shape of the safe operating space for human impacts on the Earth system}

In this paper, we define the safe operating space as those combinations of human impacts on the planetary boundaries that do not cause any planetary boundary to be transgressed, and therefore maintain the Earth system within a Holocene-like state.

To calculate the initial estimate of the safe operating space for human impacts on the Earth system in Fig. 4, we set all control variables to their planetary boundaries (all elements of $\mathbf{x}$ to 1 ). Eq. (9) shows that $\mathbf{x}$ - B $\mathbf{x}$ back-calculates the corresponding levels of total human impacts (including direct impacts and both types of human-mediated interactions).

To the explore trade-offs within the safe operating space for human impacts on the Earth system in Fig. 5, we formed two groups of planetary boundaries: land system change, freshwater use and biogeochemical flows, which all experience large impacts from agricultural activity; and climate change and ocean acidification, impacts on which are driven primarily by carbon emissions.

To simulate varying levels of agricultural activity and carbon emissions, we analysed different combinations of values for the Land-system change and Climate change elements of the direct impacts vector $\mathbf{d}$. The parallel human drivers built into the model then lead to impacts on the other planetary boundaries within those groups. We fixed the aerosol control variable at its boundary value (normalised value 1), assumed successful rehabilitation of stratospheric ozone by setting its control variable at its preindustrial value (normalised value 0 ) and assumed no direct human impacts on other planetary boundaries. We set the strength of the parallel human driver Land-system change -> Climate change to 0 to ensure that fossil fuel emissions of agricultural origin are not double-counted. We applied Eq. (8) for different combinations of $\mathbf{d}$ and counted how many control variables exceeded their boundary values and how many exceeded their zones of uncertainty.

We estimated the additional impacts on planetary boundaries resulting from two policy interventions: BECCS and a low-meat diet (see Supplementary Methods for details). For BECCS, we 
used scenarios from a global modelling study that cast its results in terms of planetary boundaries ${ }^{11}$. For a low-meat diet, we selected from a systematic review of diet change modelling ${ }^{54}$ the two studies that estimated the effects of a global transition to a vegetarian diet. For further information see Supplementary Methods. We plotted these interventions as deviations from the current direct impacts on Figure 5. Current direct impacts were estimated using Eq. (10), using the modified interaction matrices described earlier in this sub-section.

Corresponding author. Please send correspondence and requests to materials to Steven Lade, steven.lade@su.se.

Acknowledgements. The research leading to these results has received funding from the European Research Council Advanced Investigator project ERA (grant ERC-2016-ADG-743080), the Swedish Research Council Formas (grant 2014-589), the Earth League's EarthDoc program, a Royal Society University Research Fellowship, and the UK Natural Environment Research Council (grant NE/R010811/1). We thank Sarah Cornell and Rowan Trebilco for their comments on drafts of the manuscript.

Data availability. All data used in the manuscript's analyses are available in the Supplementary Information (Tables S1 and S2).

Code availability. All computations are fully described in Methods. Implementation in R of these computations is available upon request.

Competing interests. The authors declare no competing financial interests.

Author contributions. S.J.L. and W.S. conceived the study. S.J.L., W.S. and W.de V. developed the paper's methodology. All authors contributed to the survey of literature. S.J.L. performed the modelling. S.J.L. and J.F.D. contributed to graphical representation of results. S.J.L. led the writing of the paper. All authors contributed to editing the paper. 


\section{References.}

1. Steffen, W. et al. Planetary boundaries: Guiding human development on a changing planet. Science 347, 1259855 (2015).

2. Rockström, J. et al. Planetary boundaries: exploring the safe operating space for humanity. Ecol. Soc. 14, 32 (2009).

3. Downing, A. S. et al. Matching scope, purpose and uses of planetary boundaries science. Environ. Res. Lett. (2019). doi:10.1088/1748-9326/ab22c9

4. Brook, B. W., Ellis, E. C., Perring, M. P., Mackay, A. W. \& Blomqvist, L. Does the terrestrial biosphere have planetary tipping points? Trends Ecol. Evol. 28, 396-401 (2013).

5. Montoya, J. M., Donohue, I. \& Pimm, S. L. Planetary Boundaries for Biodiversity: Implausible Science, Pernicious Policies. Trends Ecol. Evol. 33, 71-73 (2018).

6. Die Bundesregierung. German Sustainable Development Strategy. (2016).

7. Griggs, D. et al. Sustainable development goals for people and planet. Nature 495, 305-307 (2013).

8. Anderies, J. M., Carpenter, S. R., Steffen, W. \& Rockström, J. The topology of non-linear global carbon dynamics: from tipping points to planetary boundaries. Environ. Res. Lett. 8, 044048 (2013).

9. Heck, V., Donges, J. F. \& Lucht, W. Collateral transgression of planetary boundaries due to climate engineering by terrestrial carbon dioxide removal. Earth Syst. Dyn. 7, 783-796 (2016).

10. Heck, V., Hoff, H., Wirsenius, S., Meyer, C. \& Kreft, H. Land use options for staying within the Planetary Boundaries - Synergies and trade-offs between global and local sustainability goals. Glob. Environ. Chang. 49, 73-84 (2018).

11. Heck, V., Gerten, D., Lucht, W. \& Popp, A. Biomass-based negative emissions difficult to reconcile with planetary boundaries. Nat. Clim. Chang. 8, 151-155 (2018).

12. Heck, V., Gerten, D., Lucht, W. \& Boysen, L. R. Is extensive terrestrial carbon dioxide removal a 'green' form of geoengineering? A global modelling study. Glob. Planet. Change 137, 123130 (2016).

13. Friedrich, J. Modeling for Planetary Boundaries: A network analysis of the representations of complex human-environmental interactions in integrated global models. (Linköping University, 2013).

14. Griggs, D., Nilsson, M., Stevance, A.-S. \& McCollum, D. A guide to SDG interactions: from science to implementation. (International Council for Science (ICSU), 2017).

15. Nilsson, M. et al. Mapping interactions between the sustainable development goals: lessons learned and ways forward. Sustain. Sci. 13, 1489-1503 (2018).

16. Pradhan, P., Costa, L., Rybski, D., Lucht, W. \& Kropp, J. P. A Systematic Study of Sustainable Development Goal (SDG) Interactions. Earth's Futur. 5, 1169-1179 (2017).

17. Jägermeyr, J., Pastor, A., Biemans, H. \& Gerten, D. Reconciling irrigated food production with environmental flows for Sustainable Development Goals implementation. Nat. Commun. 8, 15900 (2017). 
18. Independent Group of Scientists appointed by the Secretary-General. Global Sustainable Development Report 2019: The Future is Now - Science for Achieving Sustainable Development. (2019).

19. Donges, J. F. et al. Closing the loop: Reconnecting human dynamics to Earth System science. Anthr. Rev. 4, 151-157 (2017).

20. Nash, K. L. et al. Planetary boundaries for a blue planet. Nat. Ecol. Evol. 1, 1625-1634 (2017).

21. Schellnhuber, H. J. 'Earth system' analysis and the second Copernican revolution. Nature 402, C19-C23 (1999).

22. Lenton, T. Earth System Science: A Very Short Introduction. (Oxford University Press, 2016). doi:10.1093/actrade/9780198718871.001.0001

23. Hu, Y. Efficient, High-Quality Force-Directed Graph Drawing. Math. J. 10, 37-71 (2006).

24. Pasztor, J., Scharf, C. \& Schmidt, K.-U. How to govern geoengineering? Science 357, 231 (2017).

25. Foley, J. A. et al. Global consequences of land use. Science 309, 570-4 (2005).

26. Orr, S., Pittock, J., Chapagain, A. \& Dumaresq, D. Dams on the Mekong River: Lost fish protein and the implications for land and water resources. Glob. Environ. Chang. 22, 925-932 (2012).

27. Sterner, T. et al. Policy design for the Anthropocene. Nat. Sustain. 2, 14-21 (2019).

28. Rockström, J. et al. A safe operating space for humanity. Nature 461, 472-475 (2009).

29. Steffen, W. et al. Trajectories of the Earth System in the Anthropocene. Proc. Natl. Acad. Sci. U. S. A. 115, 8252-8259 (2018).

30. Rinkevich, B. Rebuilding coral reefs: does active reef restoration lead to sustainable reefs? Curr. Opin. Environ. Sustain. 7, 28-36 (2014).

31. Søndergaard, M., Jeppesen, E. \& Jensen, H. S. Lake restoration. in Encyclopedia of Lakes and Reservoirs (eds. Bengtsson, L., Herschy, R. W. \& Fairbridge, R. W.) 455-458 (Springer, 2012). doi:10.4324/9781315685977

32. Anderies, J. M., Carpenter, S. R., Steffen, W. \& Rockström, J. The topology of non-linear global carbon dynamics: From tipping points to planetary boundaries. Environ. Res. Lett. 8, 44048 (2013).

33. Clarke, L. et al. Assessing Transformation Pathways. in Climate Change 2014: Mitigation of Climate Change. Contribution of Working Group III to the Fifth Assessment Report of the Intergovernmental Panel on Climate Change (eds. Edenhofer, O. et al.) (Cambridge University Press, 2014).

34. Fajardy, M. \& Mac Dowell, N. Can BECCS deliver sustainable and resource efficient negative emissions? Energy Environ. Sci. 10, 1389-1426 (2017).

35. Harper, A. B. et al. Land-use emissions play a critical role in land-based mitigation for Paris climate targets. Nat. Commun. 9, 2938 (2018).

36. Springmann, M. et al. Options for keeping the food system within environmental limits. Nature 562, 519-525 (2018). 
37. Willett, W. et al. Food in the Anthropocene: the EAT-Lancet Commission on healthy diets from sustainable food systems. Lancet (London, England) 393, 447-492 (2019).

38. Cooper, G. S. \& Dearing, J. A. Modelling future safe and just operating spaces in regional social-ecological systems. Sci. Total Environ. 651, 2105-2117 (2019).

39. Hossain, M. S., Dearing, J. A., Eigenbrod, F. \& Johnson, F. A. Operationalizing safe operating space for regional social-ecological systems. Sci. Total Environ. 584-585, 673-682 (2017).

40. Dearing, J. A. et al. Safe and just operating spaces for regional social-ecological systems. Glob. Environ. Chang. 28, 227-238 (2014).

41. Mantyka-Pringle, C. S. et al. Climate change modifies risk of global biodiversity loss due to land-cover change. Biol. Conserv. 187, 103-111 (2015).

42. Raworth, K. A safe and just space for humanity: can we live within the doughnut. Oxfam Policy Pract. Clim. Chang. Resil. 8, 1-26 (2012).

43. Messerli, P. et al. Expansion of sustainability science needed for the SDGs. Nat. Sustain. 2, 892-894 (2019).

44. Nilsson, M., Griggs, D. \& Visbeck, M. Policy: Map the interactions between Sustainable Development Goals. Nature 534, 320-322 (2016).

45. Heistermann, M. A planetary boundary on freshwater use is misleading. Hydrol. Earth Syst. Sci. 21, 3455-3461 (2017).

46. Lewis, S. L. We must set planetary boundaries wisely. Nature 485, 417-417 (2012).

47. Galaz, V. Planetary boundaries concept is valuable. Nature 486, 191-191 (2012).

48. Rockström, J., Richardson, K., Steffen, W. \& Mace, G. Planetary Boundaries: Separating Fact from Fiction. A Response to Montoya et al. Trends Ecol. Evol. 33, 233-234 (2018).

49. Carpenter, S. R. \& Bennett, E. M. Reconsideration of the planetary boundary for phosphorus. Environ. Res. Lett. 6, 014009 (2011).

50. WMO. Executive Summary: Scientific Assessment of Ozone Depletion: 2018. (2018).

51. Engelberg, S. A Mathematical Introduction to Control Theory (Series in Electrical and Computer Engineering). 4, (Imperial College Press, 2005).

52. Anderies, J. M., Rodriguez, A. A., Janssen, M. A. \& Cifdaloz, O. Panaceas, uncertainty, and the robust control framework in sustainability science. Proc. Natl. Acad. Sci. U. S. A. 104, 15194-9 (2007).

53. Harrison, l. et al. The freshwater biodiversity crisis. Science 362, 1369 (2018).

54. Aleksandrowicz, L., Green, R., Joy, E. J. M., Smith, P. \& Haines, A. The Impacts of Dietary Change on Greenhouse Gas Emissions, Land Use, Water Use, and Health: A Systematic Review. PLoS One 11, e0165797 (2016).

55. Gu, Z., Gu, L., Eils, R., Schlesner, M. \& Brors, B. circlize implements and enhances circular visualization in R. Bioinformatics 30, 2811-2812 (2014). 
Figure 1: Conceptual framework for planetary boundaries and their interactions. A. Planetary boundary framework. A control variable for a planetary boundary quantifies human interference in the Earth system process represented by that planetary boundary. The boundary value indicates a conservative 'safe' limit for the control variable, within which the control variable remains with in the 'safe operating space' (green). Beyond the boundary value, the 'zone of uncertainty' indicates a range of increasing risk (yellow). Beyond the zone of uncertainty indicates an area of high risk to Earth system functioning (red). In the normalised units introduced in this paper, a control variable has value 0 under pre-industrial conditions and 1 at the boundary value. Interactions between planetary boundaries can affect both the boundary value and the control variable; in normalised units these effects are both captured by changes in the normalised control variable. B. Socialecological framework for interactions between the planetary boundaries. Direct human impacts on one planetary boundary (black arrows) can lead to changes in other planetary boundaries via various interaction mechanisms (orange arrows). See text for definitions of these mechanisms.

Figure 2. Interactions between the planetary boundaries. Net normalised interaction strengths between the planetary boundaries estimated by our survey. Data are as listed in Supplementary Table 2. A A circular representation of the full interaction matrix using the circlize package ${ }^{55}$ (version 0.4 .4 ) in $R$. The circumference of a circle is filled by originating and receiving ends of each interaction according to their relative strengths. Interactions where both biophysically-mediated (green) and human-mediated interactions (blue, including both reactive interactions and parallel drivers) are present are coloured a shade between blue and green according to their relative magnitudes. Black borders indicate a net negative (attenuating) link; all other links are positive (reinforcing). B A forcedirected network diagram ${ }^{23}$, which arranges nodes with stronger interactions closer together, of biophysically-mediated interactions only. Here we have re-aggregated the three biosphere integrity boundaries back into a single node (Supplementary Table 3 ). We only plot links whose strength we were able to estimate; for the full set of possible interactions that our survey identified see Supplementary Table 2.

Figure 3. The role of interactions in the current state of the planetary boundaries. Contributions of planetary boundary interactions and direct impacts to the current values of control variables. Control variables are in normalised units, where values of 0 and 1 correspond to pre-industrial conditions and the planetary boundary, respectively.

Figure 4: A safe operating space for human impacts on the Earth system. Outer circle (red): safe human impacts prior to accounting for interactions. Inner circle (black): No human impacts, i.e., preindustrial conditions. Grey bars: Safe levels of human impacts (including direct impacts, reactive human-mediated interactions and parallel human drivers) on each planetary boundary after biophysical interactions are taken into account, assuming that all other control variables are at their boundary values. Axis is in normalised units as in Fig 3. Under the assumptions made for this figure, the safe levels of human impacts on biosphere integrity are estimated by the model to be either negative (ocean and freshwater) or zero (land), due to the large impacts on biosphere integrity by other planetary boundaries.

Figure 5: Effects of interactions between planetary boundaries on the shape of the safe operating space for human impacts on the Earth system. We examined the safe operating space for the direct impacts of agriculture on land system change, biogeochemical flows and freshwater use and of carbon emissions on climate change and ocean acidification. The multiple direct impacts within each group (indicated by the parallel axis scales) are linearly co-ordinated according to our historically and globally aggregated estimates of their co-occurrence (see entries in Supplementary Table 2 and Supplementary Methods for parallel human drivers). Green region: the safe operating space, where all control variables are below their planetary boundaries. Yellow region: where at least one control variable is beyond its planetary boundary; darker shades indicate more boundaries transgressed. 
Red region: where at least one control variable is beyond its zone of uncertainty; darker shades indicate more control variables beyond their zones of uncertainty. Dashed green line: the edge of the safe operating space without interactions. The small circle indicates the current state of direct impacts on these planetary boundaries as estimated by our model. The arrows and shading indicate the trajectories following global-scale transitions to low-meat diets and BECCS. Axes are in normalised units as in Fig 3. 